\title{
Radiation Damage Studies of Lasers and Photodiodes for Use in Multi-Gb/s Optical Data Links
}

\author{
Jan Troska, Stéphane Detraz, Sarah Seif El Nasr-Storey, Pavel Stejskal, Christophe Sigaud, Csaba Soos, and \\ François Vasey
}

\begin{abstract}
Neutron and pion irradiation and annealing data from semiconductor lasers and photodiodes for use in $10 \mathrm{~Gb} / \mathrm{s}$ datalinks are presented. These components are found to be generally more radiation resistant than their older counterparts. Radiation damage in lasers has been modeled to allow extrapolation of the results obtained to the final application.
\end{abstract}

Index Terms-Laser diode, photodiode, radiation effects.

\section{INTRODUCTION}

C ERN (the European Laboratory for Particle Physics) has been operating the worlds most powerful particle accelerator-the Large Hadron Collider (LHC) [1] - since 2009. The LHC, when it reaches its design performance, will collide protons with a centre of mass energy of $14 \mathrm{TeV}$ and a peak luminosity of $10^{34} \mathrm{p} / \mathrm{cm}^{2} / \mathrm{s}$. The four experiments that have been built to exploit the physics potential of the LHC have all made use of optical data transmission technology on a very large scale, each installing tens of thousands of optical links. This technology has been a key enabler of the detector systems now in use and will continue to be so in future upgrades to the existing detectors. It is planned to upgrade the luminosity of the present LHC by a factor of ten on a timescale of ten years from the present in order to extend the statistical reach for the discovery of new and rare physics processes [2]. Such an upgrade will make the radiation environment that any components used in the detector systems must withstand even more challenging than it is at present. The radiation environment in the innermost regions at the so-called High-Luminosity (HL) or Super (S) LHC will be dominated by pions at energies in the several-hundred $\mathrm{MeV}$ range. Expressed in $1 \mathrm{MeV}$ neutrons equivalents in Silicon the expected fluences will reach $1.5 \times 10^{15} \mathrm{~cm}^{-2}$ while the total ionizing dose will reach $500 \mathrm{kGy}$ (Si) [3].

The Versatile Link project [4] is developing a bidirectional optical physical layer to enable the deployment of optical data link systems for the transmission of timing, control and read-out data to- and from the upgraded HL-LHC detectors. One aspect

Manuscript received July 22, 2011; revised October 09, 2011; accepted October 11, 2011. Date of publication November 23, 2011; date of current version December 14, 2011.

J. Troska, S. Detraz, S. S. El Nasr-Storey, C. Sigaud, C. Soos, and F. Vasey are with the CERN, CH-1211 Geneva 23, Switzerland (e-mail: jan.troska@cern.ch; Stephane.Detraz@cern.ch; sarah.storey@cern.ch; Christophe.Sigaud@cern.ch; csaba.soos@cern.ch; Francois.Vasey@cern.ch).

P. Stejskal is with the Blackett Laboratory, Imperial College, London SW7 2AZ, U.K. (e-mail: pavel.stejskal@cern.ch).

Color versions of one or more of the figures in this paper are available online at $\mathrm{http}: / /$ ieeexplore.ieee.org.

Digital Object Identifier 10.1109/TNS.2011.2172633
TABLE I

IRRADIATION TEST PARAMETERS

\begin{tabular}{lccc}
\hline \hline & $\begin{array}{c}\text { Neutrons I } \\
(\mathrm{n} . \mathrm{I})\end{array}$ & $\begin{array}{c}\text { Neutrons II } \\
(\mathrm{n} . \mathrm{II})\end{array}$ & $\begin{array}{c}\text { Pions } \\
\left(\pi^{+}\right)\end{array}$ \\
\hline Mean energy & $20 \mathrm{MeV}$ & $20 \mathrm{MeV}$ & $191 \mathrm{MeV}$ \\
Flux $\left(/ \mathrm{cm}^{2} / \mathrm{s}\right)$ & $1.5-3 \times 10^{10}$ & $1.5-3 \times 10^{10}$ & $1.5-3 \times 10^{9}$ \\
Irradiation time & $57 \mathrm{~h}$ & $24 \mathrm{~h}$ & 17 days \\
Annealing time & 6 weeks & 3 months & 2 weeks \\
\hline \hline
\end{tabular}

of the versatility of the proposed data links is the fact that both multimode transmission at $850 \mathrm{~nm}$ and singlemode transmission at $1310 \mathrm{~nm}$ will be supported, albeit by two distinct configurations of the Versatile Link. The radiation hardness of the optoelectronic components (lasers and photodiodes) that will be deployed inside the detectors in the framework of this project must be proven as these have been shown to degrade (often significantly) during particle irradiation [5]. To this end, a survey of multiple device types that are capable of operating at the target bit-rate of $4.8 \mathrm{~Gb} / \mathrm{s}$ has been carried out. Devices suitable for both $850 \mathrm{~nm}$ and $1310 \mathrm{~nm}$ operation have been studied by exposing them to both $20 \mathrm{MeV}$ neutrons and $191 \mathrm{MeV}$ pions in a series of radiation tests. Online measurements of the static characteristics of the devices both during- and after the irradiation allow a rather detailed picture of the evolution of the radiation-damage to be obtained.

Laser diodes exposed to particle irradiation show significant annealing once the particle beam is switched off. Information obtained by measuring the annealing behaviour has been incorporated into a model in order to predict the in-service degradation that will take place at much lower particle fluxes than a typical irradiation test.

\section{RADiATION TESTING}

\section{A. Devices Tested and Sources Used}

The radiation field present inside a typical LHC detector is a complex mix of many different particle types covering a very broad energy spectrum from $\mathrm{keV}$ to $\mathrm{TeV}$ depending upon the particle species [6]. The complex stoichiometry of semiconductor lasers and photodiodes makes the calculation of NonIonizing Energy Loss (NIEL) equivalence challenging, which results in a need to evaluate the radiation resistance of devices at sources that cover the range of particle types and energies found in the final application. We have therefore chosen to do testing at two radiation sources as given in Table I to cover the expected radiation field of HL-LHC.

Irradiation testing has been carried out on a broad spectrum of devices that are capable of operating at $10 \mathrm{~Gb} / \mathrm{s}$. A total of 


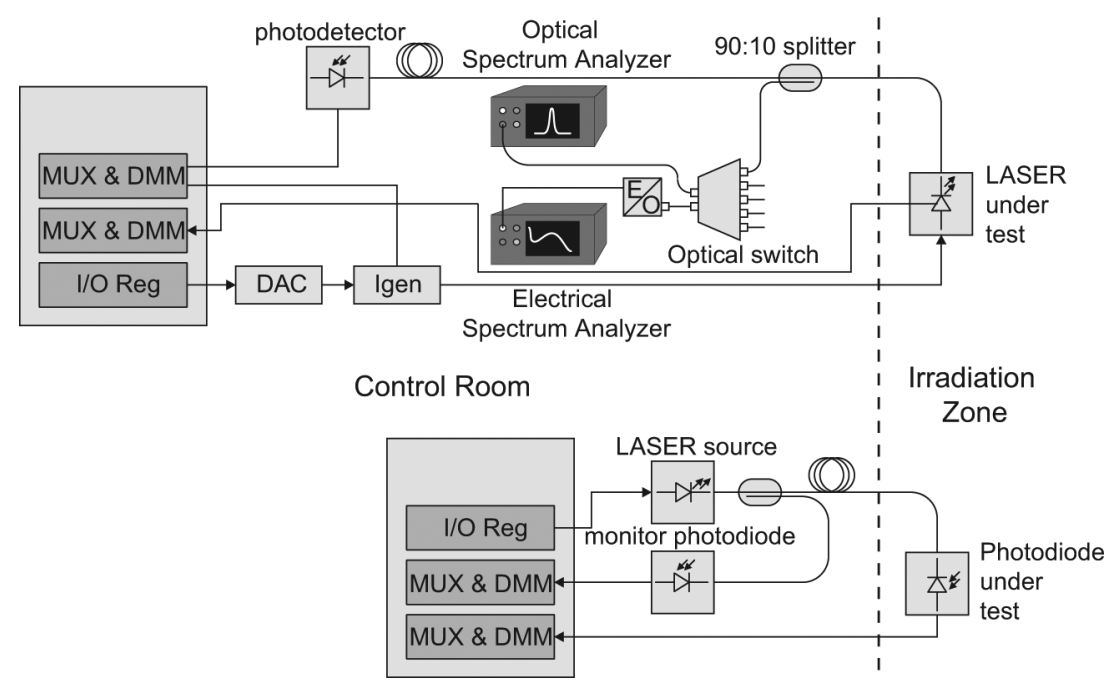

Fig. 1. Schematic of irradiation test setup showing connections between measurement instruments and lasers and photodiode DUTs.

TABLE II

LASER PARAMETERS

\begin{tabular}{lccc}
\hline \hline ID & Type & Wavelength & $\begin{array}{c}\text { Number tested } \\
\text { n.I } / \text { n.II } / \pi^{+}\end{array}$ \\
\hline A & Fabry-Pérot & $1330 \mathrm{~nm}$ & $0 / 2 / 0$ \\
B1 & Fabry-Pérot & $1310 \mathrm{~nm}$ & $3 / 2 / 2$ \\
B2 & VCSEL & $850 \mathrm{~nm}$ & $3 / 6 / 2$ \\
C1 & Fabry-Pérot & $1310 \mathrm{~nm}$ & $2 / 0 / 2$ \\
C2 & Fabry-Pérot & $1310 \mathrm{~nm}$ & $0 / 8 / 2$ \\
D & VCSEL & $1260 \mathrm{~nm}$ & $0 / 0 / 2$ \\
E & VCSEL & $1310 \mathrm{~nm}$ & $0 / 2 / 2$ \\
F1 & VCSEL & $1310 \mathrm{~nm}$ & $2 / 0 / 0$ \\
F2 & VCSEL & $1550 \mathrm{~nm}$ & $1 / 0 / 0$ \\
G & VCSEL & $850 \mathrm{~nm}$ & $3 / 4 / 2$ \\
H & Quantum Dot & $1310 \mathrm{~nm}$ & $3 / 0 / 0$ \\
\hline \hline
\end{tabular}

TABLE III

PIN PARAMETERS

\begin{tabular}{lccc}
\hline \hline ID & Type & Diameter & $\begin{array}{c}\text { Number tested } \\
\text { n.I } / \text { n.II } / \pi^{+}\end{array}$ \\
\hline $\mathrm{V}$ & InGaAs & $80 \mu \mathrm{m}$ & $2 / 0 / 2$ \\
$\mathrm{~W} 1$ & InGaAs & $80 \mu \mathrm{m}$ & $3 / 0 / 0$ \\
$\mathrm{~W} 2$ & GaAs & $70 \mu \mathrm{m}$ & $3 / 0 / 0$ \\
$\mathrm{X} 1$ & InGaAs & $30 \mu \mathrm{m}$ & $2 / 2 / 2$ \\
$\mathrm{X} 2$ & InGaAs & $60 \mu \mathrm{m}$ & $3 / 4 / 0$ \\
$\mathrm{X} 3$ & InGaAs & $80 \mu \mathrm{m}$ & $3 / 0 / 0$ \\
$\mathrm{Y} 1$ & InGaAs & $80 \mu \mathrm{m}$ & $6 / 0 / 0$ \\
$\mathrm{Y} 2$ & $\mathrm{GaAs}$ & $70 \mu \mathrm{m}$ & $3 / 0 / 0$ \\
$\mathrm{Z}$ & $\mathrm{GaAs}$ & $70 \mu \mathrm{m}$ & $2 / 0 / 2$ \\
\hline \hline
\end{tabular}

nine lasers types from eight manufacturers (Table II) and seven photodiode types from four manufacturers (Table III) have been tested. A reference laser (type C1) and photodiode (type V) from the currently operating optical link system [7] of the Compact Muon Solenoid (CMS) experiment at the LHC was also included for comparison. The devices tested cover a range of material systems suitable for $850 \mathrm{~nm}$ and $1310 \mathrm{~nm}$ operation as well as different devices structures. Both Vertical Cavity Surface Emitting Laser (VCSEL) and Edge-Emitting Laser (EEL) types were tested as well as photodiodes of different active areas. InGaAs photodiodes were tested at $1310 \mathrm{~nm}$ with singlemode fiber input and GaAs photodiodes at $850 \mathrm{~nm}$ with multimode fiber input. Not all devices were tested in both neutron and pion beams as some devices were already shown to be poor candidates after the first neutron test.

\section{B. Radiation Test Procedure}

Online measurements were performed in all tests. The test setup is shown in Fig. 1. For lasers, this consisted of measuring the light output (L) and forward voltage drop (V) as a function of drive current (I) as well as the optical and Relative Intensity Noise (RIN) spectra. For photodiodes, the output current was measured as a function of both input light level and reverse bias voltage. The cycle of measurements on all devices was typically repeated every 20 minutes throughout the exposure and annealing periods, which corresponds to a fluence step of $1.8-3.6 \times 10^{13}$ and $1.8-3.6 \times 10^{12}$ in the case of neutron and pion irradiation, respectively. Device types were typically measured sequentially and the lasers were biased some $\mathrm{mA}$ above threshold when not being measured (i.e., the level of bias increased as the irradiation proceeded and the laser threshold went up). This biasing scheme mimics the bias levels that would be used in the final application. Photodiode response was measured using a laser of fixed wavelength: $850 \mathrm{~nm}$ for the GaAs DUTs; and $1310 \mathrm{~nm}$ for the InGaAs DUTs. The photodiode reverse bias voltage was varied in $0.5 \mathrm{~V}$ steps from 0 to $2.5 \mathrm{~V}$ during the measurement and was set to $0 \mathrm{~V}$ between measurements. The ambient temperature around the DUTs was measured but not controlled during all tests and was typically around $30 \pm 5^{\circ} \mathrm{C}$. These bias and temperature conditions were the same during both irradiation and annealing periods of all tests.

\section{Photodiode Results}

Fig. 2 shows the output current of an InGaAs photodiode as a function of input light power during radiation exposure in a particle beam. The leakage- or dark-current of a photodiode is defined as the output current when the input light power is zero. As can be seen in Fig. 2, the leakage-current increases at high fluences to exceed the signal current. The responsivity $(\eta)$ of a photodiode is the amount of photo-current produced by the 


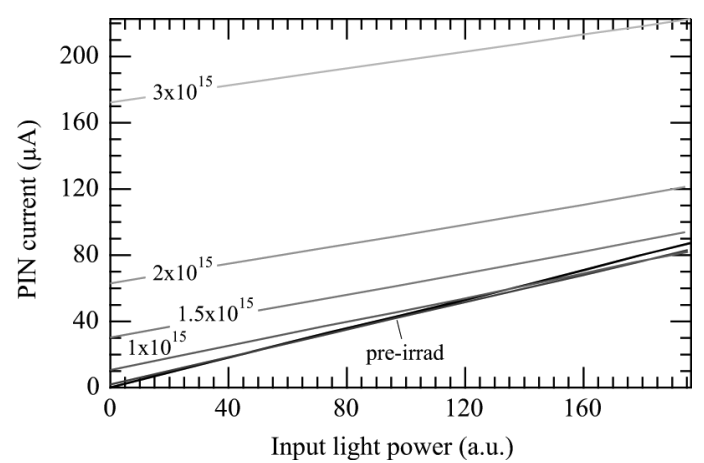

Fig. 2. Typical change in response of an InGaAs p-i-n photodiode (in this case type $\mathrm{X} 2$ at $2 \mathrm{~V}$ reverse bias) with $20 \mathrm{MeV}$ neutron fluence.

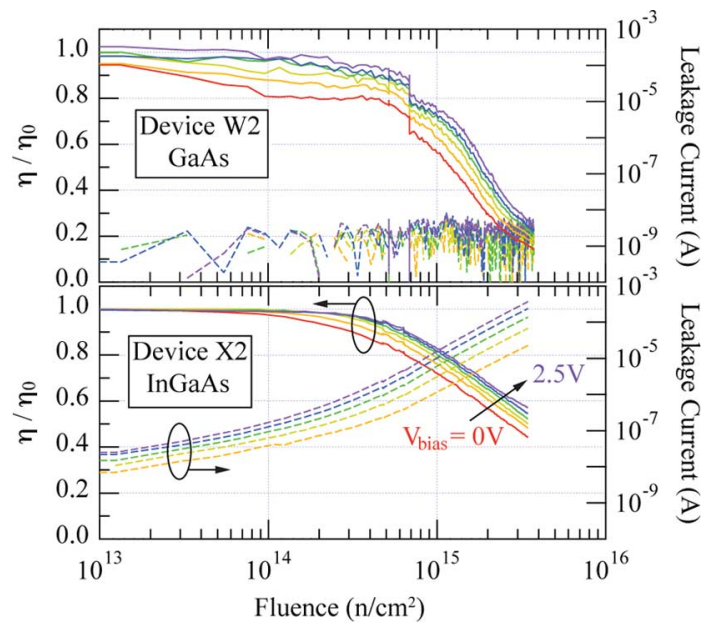

Fig. 3. Increase in leakage-current (dashed lines) and decrease in relative responsivity (solid lines) of two representative photodiodes exposed to $20 \mathrm{MeV}$ neutrons. Data are shown for increasing reverse bias voltages.

diode per unit input light power. Fig. 2 shows that the responsivity decreases at high fluence values. In order to better compare changes in responsivity between DUTs, we normalize the measured responsivity $(\eta)$ to its initial value $\left(\eta_{0}\right)$ - a quantity that we define as the relative responsivity.

The effect of irradiation on a photodiode's responsivity and its leakage (or dark) current is shown in Fig. 3 as a function of reverse bias. The two devices shown are representative of all the InGaAs and GaAs photodiodes tested. Irradiation of photodiodes causes a decrease in responsivity (the amount of photocurrent produced per unit incident optical power) in all device types tested. An overview of the increase in leakage current and decrease in responsivity of the devices tested is shown in Fig. 4. Fluences in excess of $10^{13}$ particles $/ \mathrm{cm}^{2}$ are needed in order to observe the effect and fluences beyond $10^{14}$ particles $/ \mathrm{cm}^{2}$ produce the onset of severe degradation that appears to lead to complete device failure around $10^{16}$ particles $/ \mathrm{cm}^{2}$. The unusual form of the change in responsivity of device $\mathrm{V}$ has been ascribed to the radiation-induced compensation of the lightly doped instrinsic region of the p-i-n structure [8], which is also the likely reason behind the sudden degradation observed in all device responsivities after several times $10^{14}$.

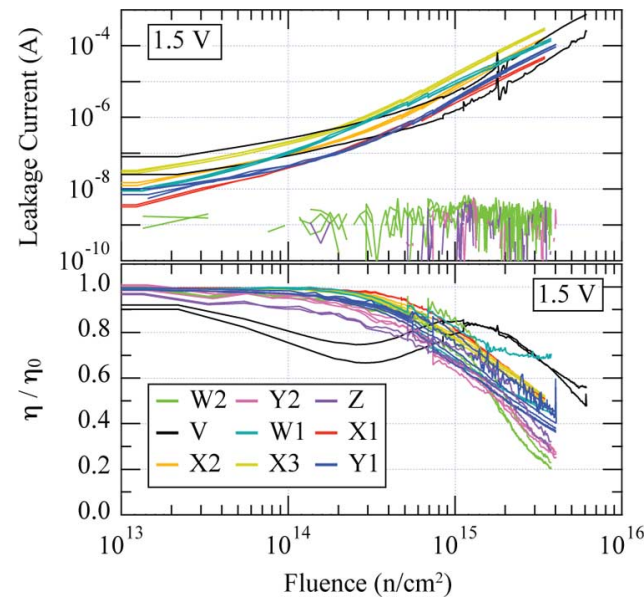

Fig. 4. Increase in leakage-current (top) and decrease in relative responsivity (bottom) of photodiodes exposed to $20 \mathrm{MeV}$ neutrons.

There is a difference in post-irradiation leakage current increase between GaAs and InGaAs photodiodes. The GaAs devices show no measurable increase in leakage current whereas the InGaAs photodiode leakage current increases by several orders of magnitude. The pre-irradiation leakage current of the GaAs devices is in fact below the sensitivity of our measurement setup, being in the tens of $\mathrm{pA}$ range. This is to be compared to the InGaAs devices where the pre-irradiation leakage current is two orders of magnitude higher, which is indicative of a higher intrinsic defect concentration in the semiconductor material. Post-irradiation leakage current increases of one order of magnitude have been reported for similar GaAs structures irradiated to similar total fluences [9], which is not incompatible with our results given the limitations of our measurement setup. The difference in magnitude of the leakage current increase between GaAs and InGaAs devices is not fully understood. The device structures are very similar so we must conclude that the GaAs material must intrinsically be less susceptible to the creation of generation type defects, which may be related to the lower instrinsic defect density in GaAs material. At the fluences of interest for HL-LHC applications the leakage current will become comparable to or even exceed the signal current, which must be taken into account in the design of the receiving amplifier.

Post-irradiation annealing has been measured to be negligible compared to the radiation-induced damage. InGaAs devices anneal a maximum of $30 \%$ of the final leakage current after hundreds of hours, compared to an initial increase of almost six orders of magnitude. Annealing of responsivity damage is not measurable. The fact that there is negligible annealing of damage in photodiodes makes it straightforward to compare the effect of pion and neutron irradiation, since no annealing means that the amount of damage observed does not depend on the particle flux during irradiation. Fig. 5 shows the results from the pion test compared to the first neutron irradiation test. In terms of leakage current, $191 \mathrm{MeV}$ pions are found to be a factor of 2-4 more damaging than $20 \mathrm{MeV}$ neutrons for the InGaAs photodiodes. The newer smaller-diameter InGaAs samples have a smaller relative damage factor than the InGaAs devices deployed in link systems of the CMS detector. These 


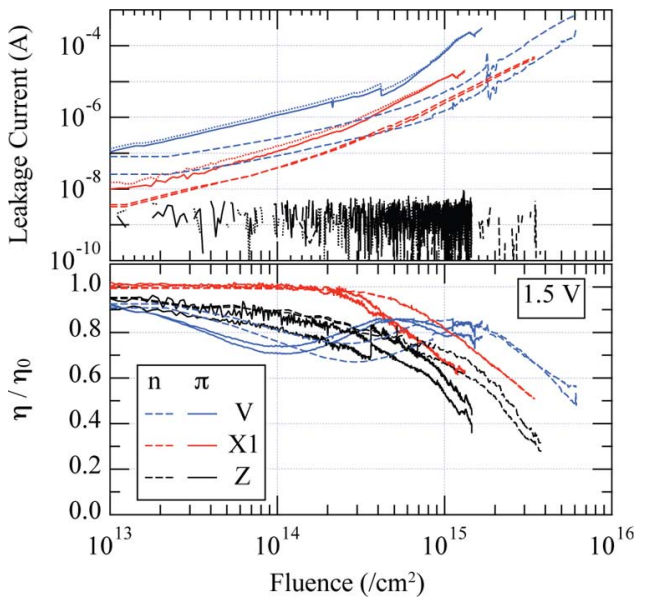

Fig. 5. Increase in leakage-current (top) and decrease in relative responsivity (bottom) of the three photodiode device types tested in both neutron (dashed lines) and pion (solid lines) beams.

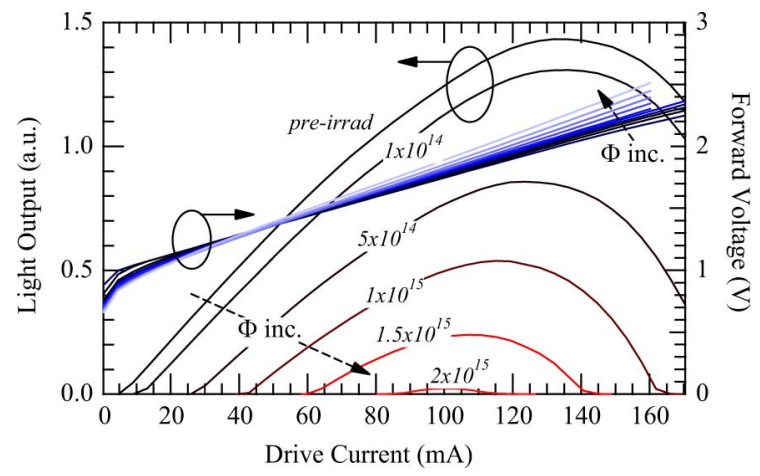

Fig. 6. Typical change in the L-I-V characteristic of an edge-emitting $1310 \mathrm{~nm}$ Fabry-Perot laser (in this case type C1) with $20 \mathrm{MeV}$ neutron fluence.

latter devices have been used as a reference in our current test programme as they have been extensively studied in the past [8]. As there is no measurable (in our test) increase in leakage current in the GaAs devices, we cannot calculate a relative damage factor for them. In terms of responsivity, $191 \mathrm{MeV}$ pions are found to be a factor of 2 more damaging than 20 $\mathrm{MeV}$ neutrons for the GaAs devices, a factor of 2.5 for the CMS InGaAs devices and a factor of 1.8 for the newer InGaAs devices. A preliminary calculation of the total Non-Ionizing Energy Loss (NIEL) has been carried out using the FLUKA [10] simulator. For comparison, the ratio of $191 \mathrm{MeV}$ pion NIEL compared to $20 \mathrm{MeV}$ neutron NIEL has been calculated to be 1.6 for GaAs and 1.8 for InGaAs. The calculated values are compatible with our measurements for the InGaAs devices but show a slight discrepancy for the GaAs devices that remains to be investigated in future tests and calculations.

\section{Laser Results}

Fig. 6 shows the typical evolution of a laser's L-I-V curves with radiation. The threshold current $\left(\mathrm{I}_{\mathrm{th}}\right)$ of a laser is defined as the drive current at which lasing operation begins (the point where the curves begin to lift-off the $\mathrm{x}$-axis in Fig. 6). From Fig. 6 it is clear to see that the threshold current increases with increasing fluence. The laser slope efficiency (E) is the amount of light output produced by the laser per unit increase in drive current in the linear output region just above $\mathrm{I}_{\mathrm{th}}$. Slope efficiency decreases with increasing fluence.

The increase of laser threshold current as a function of total fluence is shown in Fig. 7 for all devices tested. The smallest active volume devices (multimode VCSELs operating at 850 $\mathrm{nm}$ ) showed the highest resistance to radiation damage and remained functional after exposure. All of the longer wavelength singlemode devices stopped lasing at the highest fluences reached during the test. Of the singlemode devices again the smaller active volume devices (VCSELs and Quantum Dot lasers) survived to higher fluences than standard edge-emitting Fabry-Perot devices. All devices showed recovery after irradiation indicating that the lower flux exposure of the SLHC application will yield less overall damage. A similar effect was observed in terms of the damage to the slope efficiency of the lasers as shown in Fig. 8. Here we observe more easily that several devices (types B1, C1, H, F1 and F2) completely stop lasing and revert to LED-mode operation after a fluence of several $10^{15} \mathrm{n} / \mathrm{cm}^{2}$ due to the high flux used for this irradiation test. Device F2, which failed just before the beam stopped, recovered sufficiently to start lasing again during the annealing period. The other devices showed a gradual increase in their maximum light output due to spontaneous emission but did not recover sufficiently to start lasing again. The second neutron test was therefore carried out to a lower total fluence to avoid this problem and enable us to measure the annealing properties of the lasers correctly.

In order to compare the results obtained at the two irradiation sources we must take into account the annealing that occurred during the irradiation period, since the two sources were not equally bright. For this we need good information about the annealing once the source is turned off, over long timescales, so that the different flux profiles of the two sources can be modeled. The annealing data obtained over $1800 \mathrm{hrs}$ following neutron test 2 are shown in Fig. 9. We observe that the curves do not display the expected exponential form since the annealing appears to saturate before all damage is recovered as seen from a flattening of the curves in Fig. 9 for very long annealing times. We have found that the annealing data fit the Hill Equation [11] much better. The Hill equation is given by equation (1) for time $t>t_{0}$, where $\mathrm{a}, \mathrm{c}$, and $\mathrm{k}$ are fit constants

$$
I_{t h}\left(t-t_{0}\right)=I_{t h}\left(t_{0}\right)\left[1-\frac{(1-a)\left(t-t_{0}\right)^{k}}{c+\left(t-t_{0}\right)^{k}}\right] .
$$

The Hill equation, which a sigmoid function, has been very successfully applied in biochemistry in the study of reagent concentrations and differs from the exponential function by the fact that the final value at infinite time need not be zero. This means that the underlying defect concentration will never completely anneal, indicating that the equilibrium defect concentration may in fact depend upon other factors such as temperature and forward current, something that appears plausible but the magnitude of which would have to be confirmed in future tests.

Once the annealing is properly taken into account, we can scale the neutron data from test 2 to the flux profile of the pion irradiation as shown in Fig. 10. As expected, the pions were 


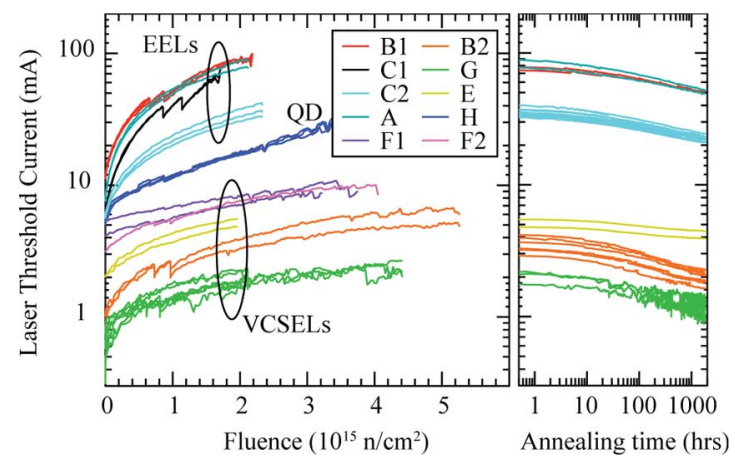

Fig. 7. Effect of irradiation and annealing on the threshold current of all lasers tested with $20 \mathrm{MeV}$ neutrons.

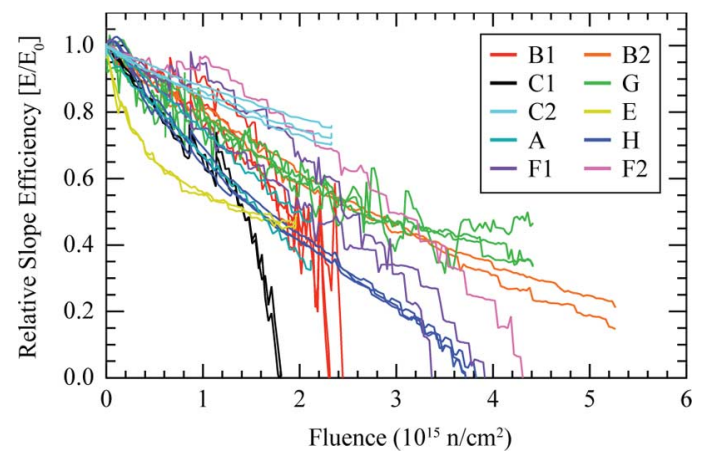

Fig. 8. Effect of irradiation on the slope efficiency of all lasers tested with 20 $\mathrm{MeV}$ neutrons. Some devices reached zero efficiency which indicates that they stopped lasing during the test.

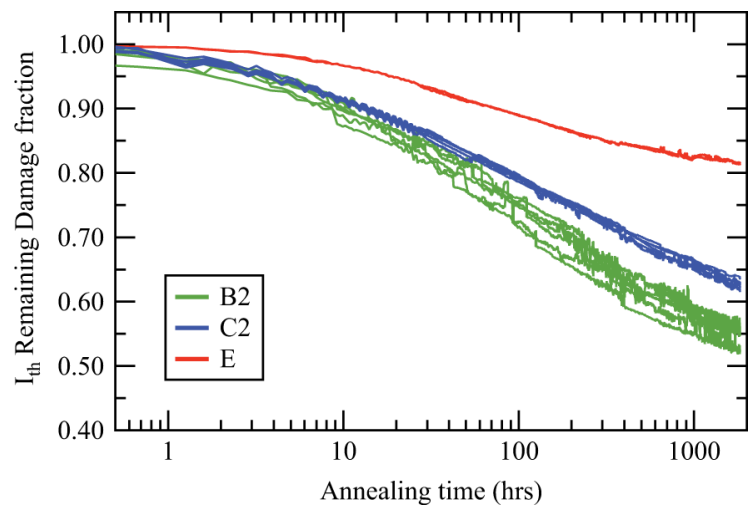

Fig. 9. Annealing of laser threshold current after neutron test 2.

found to be more damaging than $20 \mathrm{MeV}$ neutrons. We find that relative damage factors of $191 \mathrm{MeV}$ pions and $20 \mathrm{MeV}$ neutrons is 1.9 for $\mathrm{B} 2$ devices, 2.1 for $\mathrm{B} 1,2.4$ for $\mathrm{C} 2$, and 2.8 for $\mathrm{E}$. These values can again be compared to our preliminary NIEL calculations which give the equivalent ratios of 1.6 for GaAs and 1.8 for InGaAsP. Given their operating wavelength, devices B2 would be expected to follow the GaAs ratio while devices B1, $\mathrm{C} 2$, and $\mathrm{E}$ are all made from InGaAsP material. One explanation for the discrepancy between our measured ratios and the NIEL calculation is that we do not know the exact stoichiometry of the devices tested in order to make a very accurate NIEL calculation. This discrepancy will be investigated further in future work.

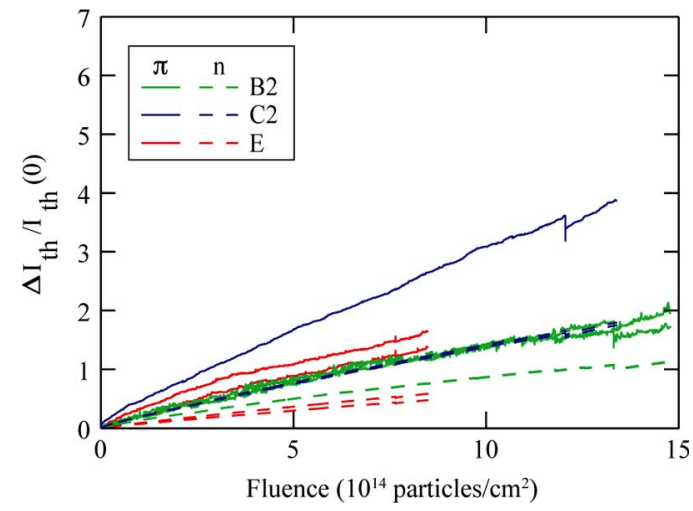

Fig. 10. Comparison of the effect of $191 \mathrm{MeV}$ pion (solid lines) and $20 \mathrm{MeV}$ neutron (dashed lines) irradiation on the increase in laser threshold current.

We have for the first time measured the Relative Intensity Noise (RIN) spectra for the lasers during irradiation. RIN spectra were measured at four bias settings above threshold as shown as an example for device type E in Fig. 11. Fitting of the data allows the extraction of some of the intrinsic characteristics of the lasers, in particular whether the modulation efficiency of the lasers remains unchanged following irradiation. The RIN spectra are first fitted using equation (2)[12] which yields the resonance $\left(f_{r}\right)$ and damping $\left(G_{d}\right)$ frequencies of the laser

$$
R I N=\frac{A+B(2 \pi f)^{2}}{16 \pi^{4}\left(f^{2}-{f_{r}}^{2}\right)^{2}+(2 \pi f)^{2}{G_{d}}^{2}} .
$$

It is instructive to introduce the following relations:

$$
\begin{array}{r}
f_{r}=D \sqrt{I-I_{t h}} \\
G_{d}=\frac{1}{2 \tau_{e}}+K f_{r}{ }^{2}
\end{array}
$$

where $P$ is the output power, $\tau_{e}$ is the differential carrier lifetime, and $D$ and $K$ are figures of merit for the high-speed response of the device under test. Furthermore, the $K$-factor can be expressed as follows[12]:

$$
K=(2 \pi)^{2}\left(\tau_{p}+\frac{\epsilon}{\nu_{g} g_{n}}\right)
$$

where $\tau_{p}, \nu_{g}$, and $g_{n}$ are the photon lifetime, the group velocity, and differential gain respectively. Fig. 12 shows the evolution of the resonance frequency of lasers of type E during irradiation. Comparison to an un-irradiated device reveals that the resonance frequency does not change appreciably during irradiation provided sufficient current is used to bias the laser after the laser threshold increases due to the irradiation. Fitting the straight line described by equation (4) to the data obtained we observe that the differential carrier lifetime above threshold decreases slightly as shown in Fig. 13. Since the differential carrier lifetime above threshold is not the same as the carrier lifetime at threshold that determines the laser's threshold current this result does not provide direct insight into to increase in threshold current, but it might provide an avenue for further investigation into the cause of the observed decrease in laser slope efficiency. The observed change in $K$-factor can be taken to indicate a decrease in the photon lifetime given the findings presented in [13] 


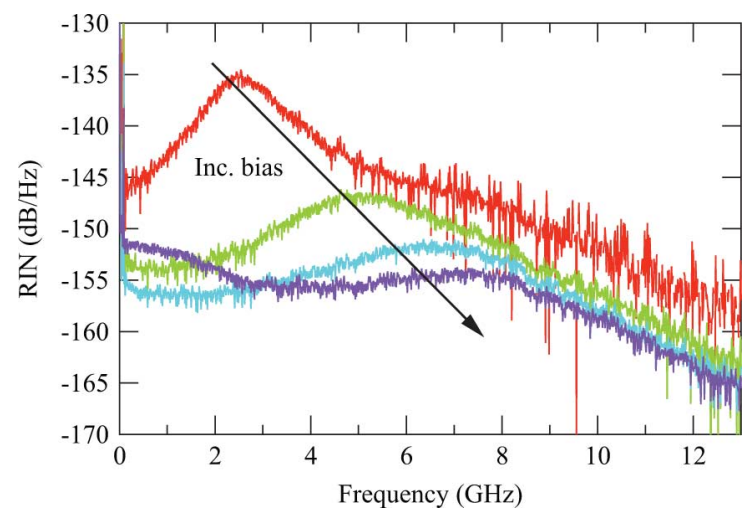

Fig. 11. Laser RIN spectra for different bias currents of a laser of type E.

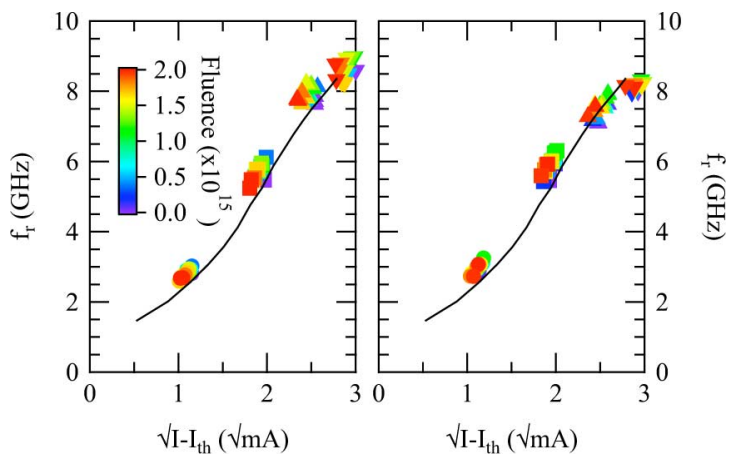

Fig. 12. Evolution of the resonance frequency as a function of bias current above threshold during $20 \mathrm{MeV}$ neutron irradiation of two lasers of type $\mathrm{E}$ (one per plot). The color-graded symbols represent the data taken as a function of neutron fluence, the four symbol types correspond to the four bias currents used. The solid line represent data taken using a finer current step size for a nonirradiated third device of the same type.

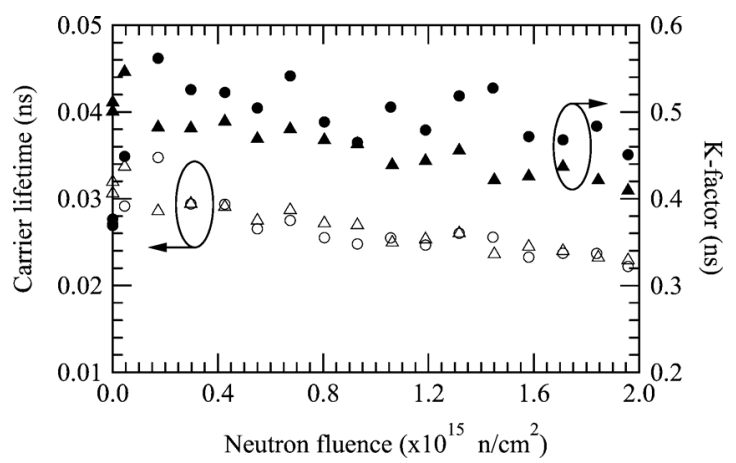

Fig. 13. Evolution of the carrier lifetime (open symbols) and K-factor (closed symbols) for the two samples of device $\mathrm{E}$ irradiated with $20 \mathrm{MeV}$ neutrons.

regarding the invariance of the differential gain of a semiconductor laser under irradiation. Although we measured the RIN spectra of several laser types during our pion and second neutron tests, the data presented were the ones to provide the most insight into changing laser parameters during irradiation. We will continue to pursue this measurement type in future tests, having improved our methods to enable us to measure both EELs and VCSELs at both 850 and $1310 \mathrm{~nm}$ wavelengths.

\section{LASER Degradation Model}

Radiation testing for validation purposes is time-consuming and expensive, a conclusion we reach based upon our experience with the quality assurance necessary when producing the 60000 lasers and 3600 photodiodes currently operating in the CMS detector at the LHC [5]. As the HL-LHC increases the radiation levels to which devices must be tested for validation purposes, this task will become even more complex in future. It would be advantageous if we could develop a method to carry out irradiation testing to lower fluences and extrapolate those results to the higher fluences of the final application. This is the motivation behind our development of a laser model that can be used to fit the irradiation data in order to be able to predict the maximum fluence at which the devices will fail. In addition, it is desirable to be able to predict the in-service degradation of the devices tested, which will be lower than the levels measured in tests that have been carried out using beam fluxes several orders of magnitude above those expected to be encountered in the final application. The degradation in semiconductor lasers will be lower because significant annealing occurs post-irradiation, indicating that damage created early in a low-flux irradiation will have annealed by the end of the irradiation as compared to a high-flux irradiation to the same total fluence. We have therefore developed a model based on laser rate equations that has been used to fit the measured data in order to extrapolate to the two conditions outlined above.

\section{A. Rate Equation Based Laser Model}

Laser operation is goverened by a set of rate equations that relate the number of photons to the number of carriers in the system. The laser rate equations for carrier density $n$ and photon number $P$ can be written as follows:

$$
\begin{aligned}
\frac{d n}{d t} & =\frac{I}{q V}-\gamma_{e}-G P \\
\frac{d P}{d t} & =G P V-\gamma P+R_{s p}
\end{aligned}
$$

where $I$ is the current, $q$ the elementary charge, $V$ the volume of the active region, $G$ is the gain per unit time and volume, $R_{s p}$ is the spontaneous emission term and $\gamma_{e}$ and $\gamma$ are the decay rates of electrons and photons, respectively. We can replace the first term of (6) with $J=I / q V$ and then $J, n$, and $P$ of (6) and (7) with dimensionless counterparts $j, N$, and $p$ to aid in fitting later. We have also introduced an additional term in the gain relation to account for the thermal rollover observed:

$$
G(n, J)=G_{0}\left(n-n_{0} J^{l}\right)
$$

where $G_{0}, n_{0}$, and $l$ are constants. Setting $l=0$ yields a linear L-I curve without thermal rollover. In the steady state that is represented by the static measurement of an L-I curve during irradiation, the rate equations can then be written in the following form:

$$
j-N-p\left(\frac{N}{1-a}-\frac{a j^{l}}{1-a}\right)=0
$$




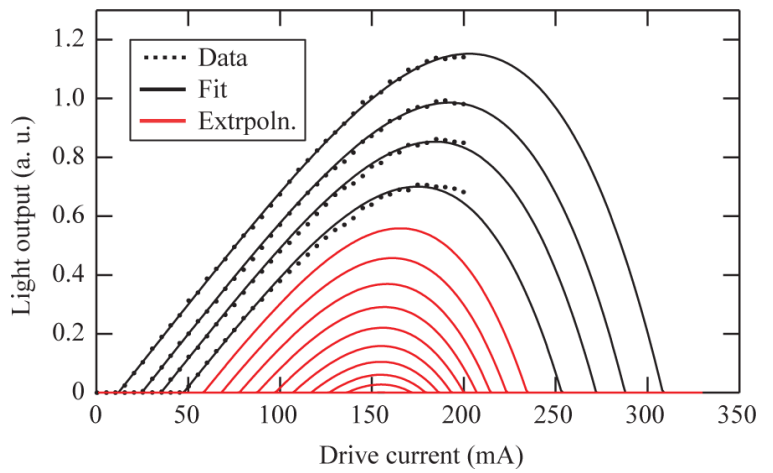

Fig. 14. Irradiation test data for laser type B1 are fitted and used to predict the further evolution to higher fluences.

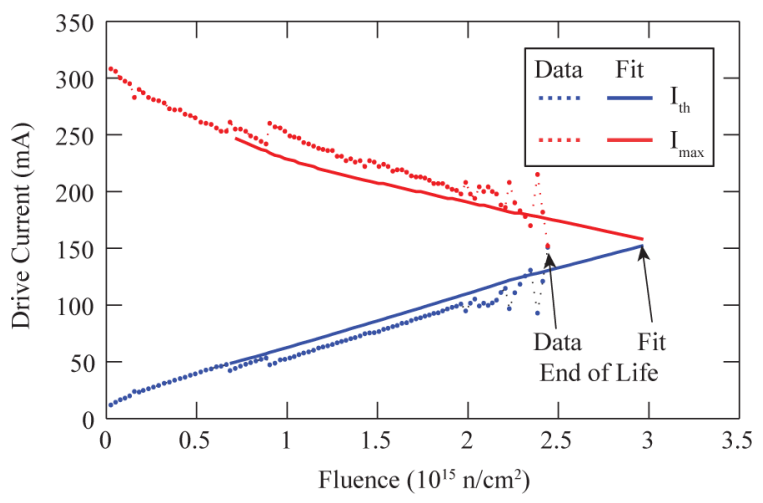

Fig. 15. Fitted evolution of the Threshold and Maximum currents of laser type B1 that are used to predict end-of-life.

$$
p\left(\frac{N}{1-a}-\frac{a j^{l}}{1-a}-1\right)+\beta_{s p} N=0
$$

where $a=n_{0} \gamma_{e} J_{t h}^{l-1}$.

\section{B. Laser End-of-Life Prediction}

We fit the pair of simultaneous equations represented by (9) and(10) to our measured L-I data (the dots in Fig. 14) and obtain the solid black lines of Fig. 14. We can then extrapolate the trends of the fit parameters in order to calculate the set of extrapolated L-I curves shown as the solid red lines in Fig. 14), which match the measured data quite well. We have defined two interesting current values for the measured and extrapolated L-I curves: the laser threshold current $I_{t h}$ where the L-I curves first lifts off the $\mathrm{x}$-axis of Fig. 14; and the maximum current $I_{\max }$ at which the L-I curve meets the same x-axis again after having rolled-over. The trend of these two points is shown in Fig. 15 as the solid lines that are to be compared to the dots representing the measured data. The agreement between data and fit is rather good and allows the point of failure where the device stops lasing to be predicted reasonably accurately at the point where $I_{t h}$ and $I_{\max }$ become equal. This model has also been used to fit the data of other laser types with similarly good results and thus paves the way for reducing the total fluence (and thus time and cost) needed to qualify devices for use in extreme radiation environments.

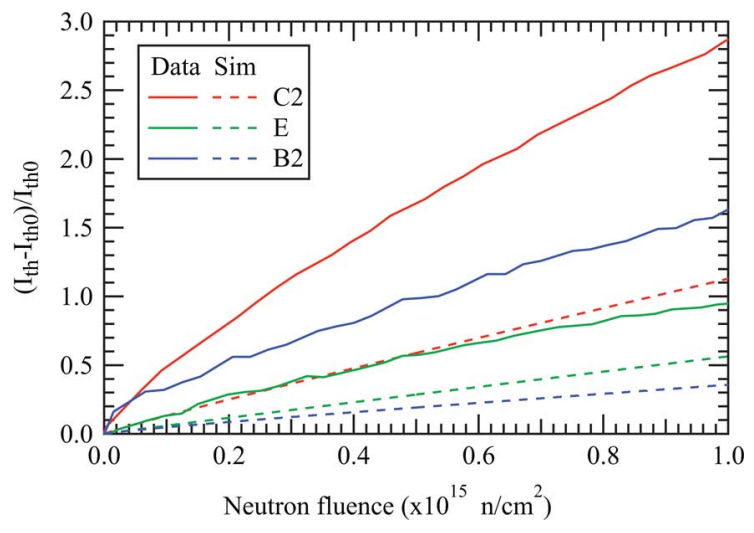

Fig. 16. Relative threshold increase measured for three device types with $20 \mathrm{MeV}$ neutrons at fluxes of $1.5-3 \times 10^{10} \mathrm{n} / \mathrm{cm}^{2} / \mathrm{s}$ and extrapolated by simulation to a lower flux of $10^{7} \mathrm{n} / \mathrm{cm}^{2} / \mathrm{s}$.

\section{System ImPACT AND Mitigation}

The L-I curve fitting and extrapolation to lower fluxes outlined in the preceding sections also allows us to predict the laser degradation for arbitrary flux profiles. This is of interest since the irradiation tests are carried out at fluxes which are 3 to 4 orders of magnitude higher than those that devices placed within the innermost HL-LHC detector regions will have to withstand. Fig. 16 shows that decreasing the flux and therefore allowing more annealing to take place during the irradiation period brings the relative increase in threshold current down by a factor of at least two. In the final application the maximum flux will be $10^{7} \mathrm{n} / \mathrm{cm}^{2} / \mathrm{s}$, so this is the value used as the worst case extrapolation of Fig. 16. The annealing conditions used, which represent the typical bias point of an operating transmitter in the final application, are most beneficial to the short-wavelength VCSEL B2. This is shown by the largest difference between the measured and simulated curves of this device relative to the others in Fig. 16.

The LHC machine does not operate continously-there are periods during which the accelerator complex is stopped to allow maintenance work to be carried out. This results in a flux profile where the radiation source is on for $2800 \mathrm{hrs}$ per year and off for the rest of the year. Taking the nominal lifetime of both LHC and HL-LHC to be ten years, we have simulated the effect of this beam profile to understand the level of laser threshold change that we have to mitigate in our system design. The result is shown in Fig. 17, where we see that we will have to mitigate increases of 10 to $20 \mathrm{~mA}$ in threshold current from a device with a pre-irradiation threshold current of 5 to 10 $\mathrm{mA}$. The Versatile Link project will use a laser driver with programmable bias current up to $45 \mathrm{~mA}$, which easily allows for such changes.

Mitigating the effect of increased leakage current in photodiodes has been done in the design of the receiving amplifier, which can sink a DC current of $1 \mathrm{~mA}$ without degradation of sensitivity to the modulated signal. The reduction in responsivity in the photodiodes will be solved at the system level by employing lasers to send signals into the detector volume that have been specially selected to have sufficient light output to mitigate the observed loss in responsivity. 


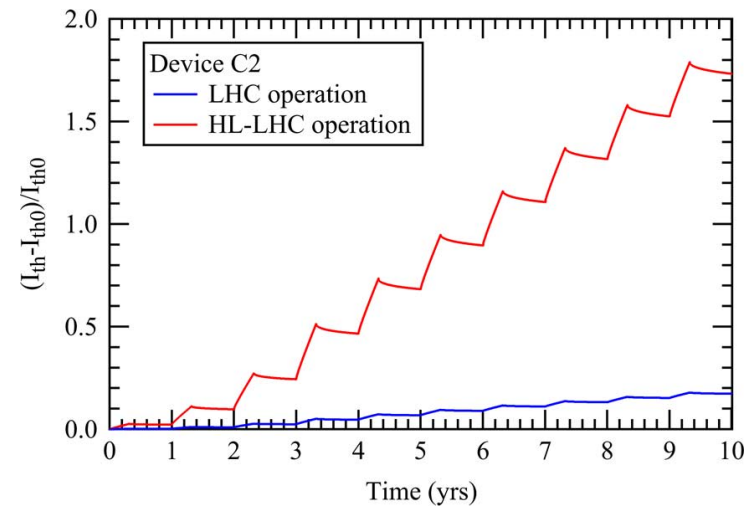

Fig. 17. Predicted relative threshold increase for operation of device type $\mathrm{C} 2$ at LHC and HL-LHC, taking into account alternating 4 months of beam on and 8 months of beam off.

\section{CONCLUSION}

A large spectrum of lasers and photodiodes has been irradiated with both neutrons and pions in order to assess their suitability for use in future data link systems to be deployed in particle physics detector systems. Encouragingly, devices from many different manufacturers appear to be sufficiently radiation resistant for use in optical links for use at HL-LHC. We find that laser devices with smaller active volumes (VCSELs) are more radiation resistant than edge-emitting laser structures. More modern edge-emitting lasers designed for $10 \mathrm{~Gb} / \mathrm{s}$ operation (types $\mathrm{A}, \mathrm{B} 1$, and $\mathrm{C} 2$ ) are typically more radiation resistant that the devices deployed in our current optical link systems (type C1). GaAs photodiodes are found not to suffer from the same dramatic increase in leakage current as their InGaAs counterparts, whereas the former do appear to degrade faster in terms of their loss of responsivity.

Our comparison of different irradiation sources shows that $191 \mathrm{MeV}$ pions are a factor of 2 to 3 more damaging than 20 $\mathrm{MeV}$ neutrons. This will allow us to concentrate future testing efforts at the $20 \mathrm{MeV}$ neutron source which is significantly easier to access and use than the pion beam. We have directly measured the degradation of the carrier lifetime in semiconductor lasers for the first time using an online measurement of laser RIN. The RIN measurements also show that there is no degradation in modulation speed with irradiation, which has been a concern for some time since we are only able to make static measurements during irradiation testing. A degradation model based on laser rate equations has been developed which permits the prediction of a laser's end-of-life at high fluence from low fluence data. This will allow shorter in-beam testing times in future, yielding important savings in terms of test time and cost.

The radiation damage observed in the devices can be fully mitigated at the system-level by using a variety of techniques. The laser driver is able to supply sufficient current to mitigate increases in laser threshold current that have been predicted to be limited to $20 \mathrm{~mA}$ in the worst case. The receiving amplifier has been designed to sink a leakage current of up to $1 \mathrm{~mA}$ without adversely affecting the dynamic performance. Finally, in the control rooms we will deploy lasers with sufficiently high output power to overcome the observed $50 \%$ to $70 \%$ decrease in responsivity of the photodiodes.

\section{ACKNOWLEDGMENT}

The authors would like to thank our collaborators from with the Versatile Link project for many fruitful discussions on the topics covered in this paper. They would also like to thank O. Militaru of UC Louvain and the team of operators at the cyclotron there as well as M. Glaser of CERN for their help in the preparation and execution of the neutron and pion tests, respectively.

\section{REFERENCES}

[1] CERN. The Large Hadron Collider [Online]. Available: http:// cern.ch/lhc

[2] N. Hessey, E. Dho and F. Vasey, Eds., "Overview and electronics needs of ATLAS and CMS high luminosity upgrades," in Proc. Topical Workshop Electron. Particle Phys., 2008, pp. 323-327.

[3] K. Gan, K. Gill, J. Troska, F. Vasey, T. Huffman, C. Issever, T. Weidberg, J. Ye, P. Skubic, R. Boyd, and F. Rizatdinova, "Joint ATLAS-CMS Working Group on Optoelectronics for SLHC Report From Sub-Group B," [Online]. Available: https://edms.cern.ch/document $/ 882783 / 2.6$

[4] L. Amaral, S. Dris, A. Gerardin, T. Huffman, C. Issever, A. J. Pacheco, M. Jones, S. Kwan, S.-C. Lee, Z. Liang, T. Liu, Z. Meng, A. Prosser, S. Padadopoulos, I. Papakonstanstinou, C. Sigaud, S. Silva, C. Soos, P. Stejskal, J. Troska, F. Vasey, P. Vichoudis, T. Weidberg, A. Xiang, and J. Ye, "The versatile link, a common project for super-LHC," $J$. Instrum., vol. 4, no. 12, p. 12003, 2009.

[5] K. Gill, M. Axer, S. Dris, R. Grabit, R. Macias-Jareno, E. Noah, J. Troska, and F. Vasey, "High Statistics Testing of Radiation Hardness and Reliability of Lasers and Photodiodes," in Proc. 10th Workshop Electron. LHC Future Exper., 2004, pp. 153-157 [Online]. Available: http://cdsweb.cern.ch/search?sysno=002443931CER

[6] The CMS collaboration, CMS Tracker Tech. Design Rep. CERN, vol. CERN-LHCC-98-006 1998 [Online]. Available: http://cdsweb. cern.ch/record $/ 368412 ?$ ln $=$ en

[7] J. Troska, G. Cervelli, F. Faccio, K. Gill, R. Grabit, A. Sandvik, F. Vasey, and A. Zanet, "Optical readout and control systems for the CMS tracker," in Proc. IEEE Nucl. Sci. Symp., Nov. 2002, vol. 1, pp. 233-237.

[8] K. Gill, M. Axer, S. Dris, R. Grabit, R. Macias, E. Noah, J. Troska, and F. Vasey, "Radiation hardness assurance and reliability testing of InGaAs photodiodes for optical control links for the CMS experiment," IEEE Trans. Nucl. Sci., vol. 52, no. 5, pp. 1480-1487, Oct. 2005.

[9] E. L. Blansett, D. K. Serkland, M. J. Cich, K. M. Geib, G. M. Peake, R. M. Fleming, D. L. Wrobel, and T. F. Wrobel, "Final report on ldrd project 105967: exploring the increase in GaAs photodiode responsivity with increased neutron fluence," Sandia National Laboratories 2008, Tech. Rep. SAND2007-8095.

[10] A. Fasso', A. Ferrari, S. Roesler, J. Ranft, P. R. Sala, G. Battistoni, M. Campanella, F. Cerutti, L. De Biaggi, E. Gadioli, M. V. Garzelli, F. Ballarini, A. Ottolenghi, D. Scannicchio, M. Carboni, M. Pelliccioni, R. Villari, V. Andersen, A. Empl, K. Lee, L. Pinsky, T. N. Wilson, and N. Zapp, The FLUKA code: Present applications and future developments. : ArXiv Physics e-prints, 2003.

[11] A. V. Hill, "The possible effects of the aggregation of the molecules of haemoglobin on its dissociation curves," J. Physiol., vol. 40, pp. I-VII, 1910.

[12] M. Tatham, I. Lealman, C. Seltzer, L. Westbrook, and D. Cooper, "Resonance frequency, damping, and differential gain in $1.5 \mu \mathrm{m}$ multiple quantum-well lasers," IEEE J. Quantum Electron., vol. 28, no. 2, pp. 408-414, Feb. 1992.

[13] M. Boutillier, O. Gauthier-Lafaye, S. Bonnefont, F. Lozes-Dupuy, D. Lagarde, L. Lombez, X. Marie, V. Ligeret, O. Parillaud, M. Krakowski, and $\mathrm{O}$. Gilard, "Measurement of irradiation impact on carrier lifetime in a quantum well laser diode," IEEE Trans. Nucl. Sci., vol. 56, no. 4, pp. 2155-2159, Aug. 2009. 